\title{
Locus of semantic priming effects in speeded naming
}

\author{
ALAN H. KAWAMOTO, KATHRYN GOELTZ, JENNIFER T. AGBAYANI, and KRISTY GROEL \\ University of Califormia, Santa Cruz, Califormia
}

\begin{abstract}
The locus of semantic priming effects was examined by measuring onset and rime durations as well as response latencies of words with consistent and inconsistent pronunciations, using the postvocalic naming task. We found that the effect of a semantic prime on naming duration was localized rather than spread across the entire word; onset durations were shorter in the related condition than in the unrelated condition, but rime durations were equal in the two prime conditions. Moreover, the priming effect on onset durations was larger for words with inconsistent than for those with consistent pronunciations. These duration results cannot be accounted for by previous proposals, but they can be accounted for by models in which phonemes are activated in parallel rather than serially from left to right and in which motor programs are based on phonemes rather than syllables. Contrary to previous reports of an interaction of prime and regularity (a factor closely related to consistency) on naming latency, we found no interaction of prime and consistency on response latency. We argue that this conflict is only apparent and arises because naming latency conflates response latency and initial phoneme duration for some targets.
\end{abstract}

The goal of psycholinguistics is to determine the representations, the architectures, and the processes involved in natural language comprehension and production. One approach to achieving this goal is to determine the loci of processing difficulties. To do so, stimuli varying along one or more dimensions are compared in terms of the time needed to complete processing. One of the most commonly used dependent variables in assessing processing difficulty is response latency. However, response latency can only assess processing difficulties that arise before a response has been initiated. To assess processing difficulties that arise after a response has been initiated, response $d u$ ration must be assessed.

In this study, we focus on the effects of semantic priming on naming duration. To date, a number of studies have demonstrated that the duration of a word read aloud decreases when preceded by an appropriate context. In some cases, the context consisted of a sentence (Fowler \& Housum, 1987; Lieberman, 1963; Shields \& Balota, 1991), and in other cases, it consisted of just a single word (Balota, Boland, \& Shields, 1989). In the studies of context effects on duration cited above, the target word was spoken together with the context when the context consisted of a sentence or was spoken by itself when the context consisted of a single word.

The present study follows up on the study by Balota et al. (1989) in that we consider a single target word read aloud in the standard semantic priming paradigm. The primary goal of our study is to determine whether the ef-

This research was supported by grants from the Academic Senate and the Social Sciences Division of the University of California at Santa Cruz. We thank Chris Kello for his assistance on various aspects of the project. Correspondence concerning this article should be addressed to A. H. Kawamoto, Department of Psychology. University of California, Santa Cruz, CA 95064 (e-mail: ahk (acats.uesc.edu). fect of a semantic prime on duration is localized to part of a word or spread across the entire word. In particular, we will determine whether there are differences in the duration of the onset, the consonants preceding the vowel in a syllable, and the duration of the rime, the vowel and the consonants following the vowel in a syllable. A secondary goal is to investigate the interaction of prime and the spelling-to-pronunciation consistency of the target word. In particular, we consider whether a semantic prime has a different effect on consistent than on inconsistent wordswords whose pronunciations are consistent or inconsistent, respectively, with the majority of words with the same body (i.e., words with identical spellings of the vowel and following consonants).

\section{Locus of Semantic Priming Effects}

Semantic priming effects on naming duration can arise in at least two possible stages of the naming process during the pronunciation assembly stage or after the pronunciation assembly stage. We begin by considering how onset and rime duration effects could arise during the pronunciation generation stage by considering two specific sequences in which phonemes are activated-in parallel or in sequence from left to right. This discussion of the parallel and sequential phoneme activation schemes is based on two specific models, the conspiracy model (Taraban \& McClelland, 1987) and Dell's (1986, 1988) speech production model, respectively.

In addition to the two possible phoneme activation sequences, two possible motor programs, one based on the phoneme and the other based on the syllable, will be considered. For the phoneme-based motor program, each successive phoneme in the word, starting with the initial phoneme, is articulated as soon as it reaches threshold and continues to be articulated until the subsequent phoneme reaches threshold. (This assumption corresponds 
to the initial-phoneme criterion discussed in Kawamoto, Kello, Jones, \& Bame, 1998). Thus, differences will arise in the duration of a particular phoneme if there are differences in when the following phoneme reaches threshold. For the syllable-based motor program, articulation is initiated only after all the phonemes in the syllable reach threshold. (This assumption corresponds to the whole word criterion discussed in Kawamoto et al.) Thus, no differences will arise in the duration of a particular phoneme, because the following phoneme is available when articulation is initiated. Note that the motor program adopted is completely orthogonal to other assumptions made by a model (e.g., sequential vs. parallel phoneme activation, local vs. distributed representation of a word, or body- vs. grapheme-based measures of pronunciation).

The first account arising during the pronunciation assembly stage that we consider assumes parallel activation of phonemes. In the parallel model, there is a lexical level in which every word is represented by a single unit. These lexical units form reciprocal connections with units representing position-based letters, units representing positionbased phonemes, and units representing semantic features. To generate a pronunciation, letters in the target activate corresponding letter units, which, in turn, activate lexical units. All the lexical units corresponding to words that have spellings similar to the target are activated, and these lexical units activate their corresponding phonemes in parallel. For low- (but not high-) frequency words, the activation of a particular phoneme will increase as the probability of a given grapheme-to-phoneme mapping increases. Only low-frequency targets are affected by lexical units of similarly spelled words, because their resting levels are low; the higher resting levels of highfrequency words confer some immunity against the effects of similarly spelled words. This difference between the resting levels of high- and low-frequency words can thus account for the lack of a consistency effect on naming latency for high-frequency words (see the summary in Seidenberg \& McClelland, 1989) and a smaller semantic priming effect on naming latency for high-frequency words than on that for low-frequency words (Becker, 1979). Consequently, we restrict our attention to targets that are low in frequency, because effects of priming on naming duration are expected to be largest for lowfrequency words.

The putative time courses of activation of the phonemes in the unrelated condition for a matched pair of lowfrequency inconsistent and consistent words such as pint and pond, respectively, are shown at the top of Figure 1. (For the targets that we consider, consistency is determined by the vowel, and the vowel is always preceded by a single consonant.) The phonemes become activated at different rates; consonants become activated more quickly than vowels, and the vowels of consistent words, such as pond, become activated more quickly than the vowels of inconsistent words, such as pint. Note that the differen-
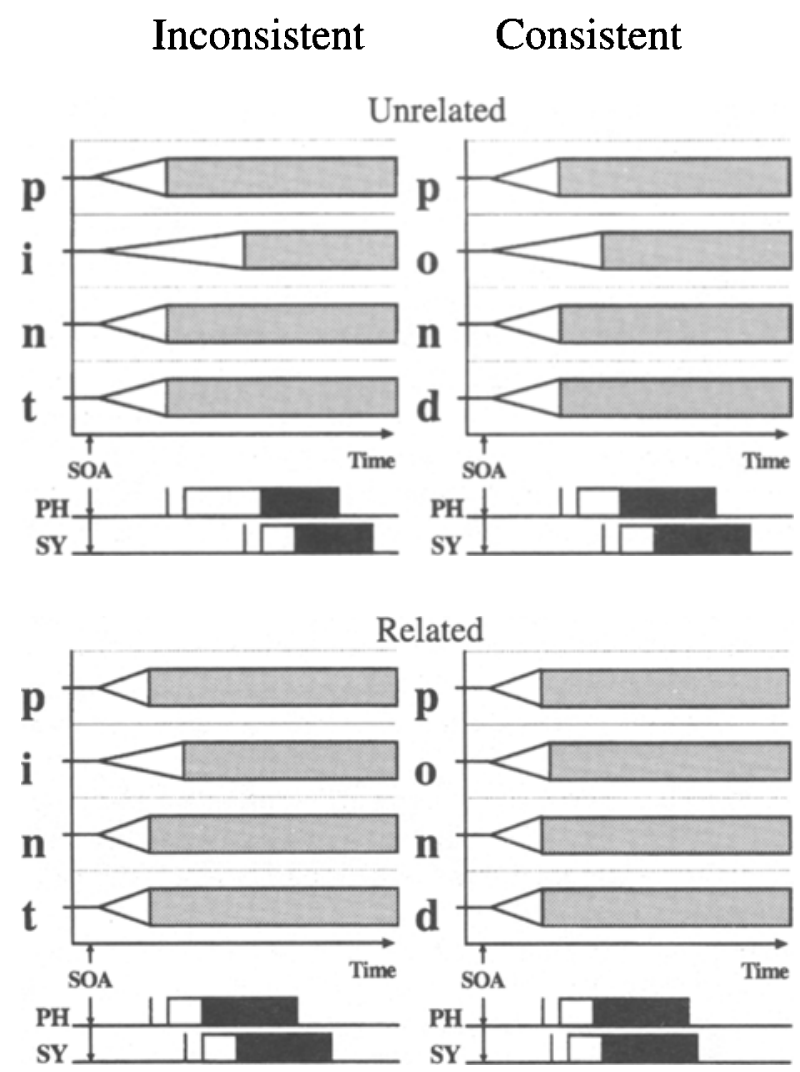

Figure 1. Putative time course of activation of phonemes of consistent and inconsistent words. The consonants are activated more quickly than the vowels, and vowels from consistent words are activated more quickly than vowels from inconsistent words. Phonemes are activated more quickly in the related condition than in the unrelated condition. Below the set of activation curves are "articulation" bars assuming a phoneme-based (PH) or syllablebased (SY) motor program. On each articulation bar, the light and dark sections correspond to articulation of the onset and rime, respectively.

tial activation of consonants and vowels leads to threshold being reached by the onset and the vowel (but not by the coda, the consonants following the vowel) in succession, despite the fact that sequential activation of the slots was not explicitly incorporated into the model.

To account for semantic priming effects, units corresponding to semantic features are needed. There are reciprocal connections between semantic features and lexical units. When the target is preceded by a semantically related prime, semantic features will also activate the lexical unit corresponding to the target, but when the target is preceded by a semantically unrelated prime, semantic features will not activate the lexical unit corresponding to the target. Thus, in the related prime condition, all the phoneme units will reach threshold sooner than they will in the unrelated condition, with vowels being facilitated more than consonants, and vowels of inconsistent words being facilitated more than vowels of consistent words (see bottom of Figure 1). Note that the time courses of the 
vowels still lag slightly behind those of the consonants, although they do not necessarily have to; the time course of activation of the consonants relative to the vowels depends on how inconsistent the pronunciation of the target is and how strongly related the semantic prime is to the target.

The response latency as well as the onset and rime duration predictions for each condition are depicted graphically on the two articulation bars below the corresponding set of phoneme activation curves in Figure 1. These articulation bars are on the same time scale as the phoneme activation curves. The first articulation bar (labeled "PH") corresponds to the predictions of a phoneme-based motor program, and the second articulation bar (labeled "SY") corresponds to the predictions of a syllable-based motor program. The vertical segment on each articulation bar corresponds to the point in time at which the criterion to initiate articulation is reached. The light section corresponds to the period during which the onset is being articulated, and the dark section corresponds to the period during which the rime is being articulated. Response latency, which we define as the point in time at which articulation is initiated, thus corresponds to the time from the presentation of the target to the left edge of the light section. Note that our definition of response latency does not correspond exactly to naming latency, the latency of the onset of acoustic energy in the naming task. This distinction is particularly important for words beginning with certain consonants, as will be discussed in more detail in the next section. Also note that there is a delay between the time at which criterion is reached and the time at which articulation is initiated. This delay arises because of the time required to retrieve the correct motor program and to activate the appropriate speech articulators.

The first set of onset and rime duration predictions for the parallel phoneme activation scheme assumes a phoneme-based motor program. The most important predictions, at least with respect to the locus of semantic priming effects on naming duration, are that (1) onset durations are shorter in the related condition than in the unrelated condition and (2) rime durations are equally long in the related and unrelated conditions. We also note that the effect of priming on onset duration is larger for inconsistent words than for consistent words. The effect of a related prime on onset duration arises because the vowel is activated more quickly relative to the onset in the related condition than in the unrelated condition. Thus, the onset duration, the interval between the initiation of the first onset consonant and the vowel, is shorter in the related condition. By contrast, there is no effect of prime on rime duration, because, by the time articulation of the vowel is initiated, all the consonants in the coda have already reached threshold.

The second set of predictions assumes a syllable-based motor program. The most important predictions are that (1) onset durations are equally long in the related condition and in the unrelated condition and (2) rime durations are equally long in the related and unrelated conditions.
In fact, the onset durations in all four conditions are identical, and the rime durations in all four conditions are identical. This pattern of duration results arises because all the phonemes of a word are available when articulation is initiated and, thus, no local processing difficulty leading to longer phoneme durations will be encountered.

The second account arising during the pronunciation generation stage that we consider assumes sequential activation of phonemes. The sequential account is similar to the parallel account, with one important difference: there are word-shape header units, units representing the phoneme categories for a word (e.g., CV, CVC), that lead to sequential (left-to-right) activation of the slots (Dell, $1986,1988)$. When the target is presented, letter units as well as semantic units activate the lexical units, which, in turn, simultaneously activate the phonemes and the wordshape header. The word-shape header is important because it assigns a word's phonemes to the slots sequentially from left to right by differentially activating the slots when a pronunciation is being assembled. Balota et al. (1989) adapted Dell's model to account for the temporal effects involved in semantic priming by assuming that the time between successive phonemes of a word reaching some threshold is shorter with a semantically related prime than with an unrelated prime.

As in the parallel account, there are two sets of predictions for the serial account, depending on which motor program is assumed. If a phoneme-based motor program is assumed, then (1) the onset duration is shorter in the related condition than in the unrelated condition, and (2) the rime duration is shorter in the related condition than in the unrelated condition. We note that this particular proposal was one of the two proposals put forth by Balota et al. (1989) to account for shorter whole word durations in the related condition than in the unrelated condition. By contrast, if a syllable-based motor program is assumed, then (1) the onset durations are identical in the related and unrelated conditions, and (2) the rime durations are identical in the related and unrelated conditions.

In addition to the possibility of duration differences arising during the pronunciation assembly stage, duration differences could also arise after the complete pronunciation of a word has been assembled. One account, first proposed by Balota et al. (1989), is based on cooperative principles of language production (Grice, 1975). According to these Gricean cooperative principles, a speaker takes the listener into consideration when speaking. Because the target in the related condition is more predictable, less stimulus information needs to be communicated. Indeed, the duration as well as the relative peak amplitudes of target words are smaller in a context that is highly predictable than in a context that is unpredictable (Lieberman, 1963). If duration differences arise because of such cooperative principles, then (1) onset durations are shorter in the related condition than in the unrelated condition, and (2) rime durations are shorter in the related condition than in the unrelated condition. Thus, the two accounts previously proposed by Balota et al., the cooperative account 
and the sequential phoneme activation with phonemebased motor program account, both make the same predictions for onset and rime durations in the standard semantic priming paradigm.

All together, there are three different patterns of duration effects that are predicted: (1) shorter onset durations in the related condition than in the unrelated condition, but equal rime durations in the two conditions; (2) equal onset and rime durations in the two conditions; and (3) shorter onset and rime durations in the related condition than in the unrelated condition. Because naming duration differences for the entire word have already been reported at the level of the word (Balota et al. 1989), the second set of predictions can be eliminated at this point. However, there is still the issue of whether duration differences are localized to the onset or found across the entire word (predictions 1 and 3, respectively). The experiment was designed to adjudicate between models that make these differential predictions.

\section{Measuring Onset and Rime Durations}

To test the models, the durations of the onset and the rime must be determined. However, measuring the durations of the onset and rime is nontrivial in general, because the boundary between the onset and the rime is usually marked only by subtle differences in acoustic characteristics, rather than by the simple presence or absence of acoustic energy. By contrast, the measurement of the duration of isolated words is relatively easy, because the beginning and ending of a word are usually marked by the transition from the absence to the presence of acoustic energy and vice versa, respectively. Fortunately, there is a class of phonemes, the plosives $(/ \mathrm{p} /, / \mathrm{t} /$, $/ \mathrm{k} /, / \mathrm{b} /, / \mathrm{d} /, / \mathrm{g} /$ ), for which the boundary is easy to determine because plosives, especially unvoiced plosives, are silent until the end of the phoneme, at which time there is an explosive release of pressure. However, this acoustic property of plosives that allows their end to be determined easily also means that the duration of a word-initial plosive cannot be determined acoustically when the standard naming task is used, because the initial silent portion of the word-initial plosive cannot be distinguished from the silence preceding the response.

One possible solution for determining onset durations for words beginning with a single plosive consonant is to use the postvocalic naming task introduced previously (Kawamoto et al., 1998). In the postvocalic naming task, subjects vocalize (i.e., say "uuhhh") before the target word appears and continue to vocalize until they begin naming the target. For words beginning with plosives, the beginning of the plosive corresponds to the transition from acoustic energy generated by the "uuhhh" to silence, and the end of the plosive corresponds to the transition from silence to acoustic energy generated by the release of the plosive. The response latency corresponds to the latency of the vocalic offset; the onset duration corresponds to the duration of the silent gap; and the rime duration corresponds to the duration of the remainder of the utterance. Thus, response latency, onset duration, and rime duration can all be determined by finding the boundary between the presence and the absence of acoustic energy.

For the postvocalic naming task, we assume that the offset of the initial "uuhhh" corresponds to the initiation of a word-initial plosive consonant. However, the offset of the "uuhhh" might simply be a response to all targets, not only to those targets that begin with plosive consonants. If this were the case, there would be a silent gap before every word, including words beginning with nonplosive consonants. To determine whether this was the case, a control study had previously been carried out (Kawamoto et al., 1998). In that study, there were some words beginning with nonplosive consonants for which no silent gap was found for all subjects. Thus, the offset of the "uuhhh" in the postvocalic naming task does correspond to the beginning of the plosive, and the vocalic offset latency measure is a valid measure of response latency for words beginning with plosives.

\section{METHOD}

\section{Subjects}

Sixty University of California at Santa Cruz undergraduate students participated in the experiment to fulfill a psychology class requirement. All the subjects were native English speakers who were unaware of the purpose of the experiment and had normal or corrected-to-normal vision.

\section{Stimuli}

The targets, shown in the Appendix together with their primes, consisted of 14 pairs of low-frequency consistent and inconsistent monosyllabic words matched on initial phoneme. In addition, the means for the following variables were closely matched across the consistency condition: For consistent targets, as compared with inconsistent targets, mean printed word frequency (Kučera \& Francis, 1967) was 10.57 , as compared with 9.00 ; mean summed bigram frequency was 4.527 .5 , as compared with $5,247.86$; number of letters was 4.07 , as compared with 4.14 , and number of phonemes was 3.29 , as compared with 3.21 . All targets had onsets consisting of a single plosive. None of the targets had the same body, nor did any target rhyme with another target.

Related and unrelated primes were generated for each target word. The related prime was either semantically or associatively related to the target. The related and unrelated primes were matched on initial phoneme and number of syllables, and they had roughly the same number of letters and phonemes. Twenty different subjects rated the prime-target pairs for relatedness on a 7-point scale. The means of the relatedconsistent pairs, the related-inconsistent pairs, the unrelated-consistent pairs, and the unrelated-inconsistent pairs were 5.6, 5.1, 1.8, and 1.8, respectively.

Two counterbalanced lists were generated, with half of the subjects seeing the first list and the other half seeing the second list. In the first list, half of the consistent-inconsistent target pairs were preceded by a related prime, and the remaining half of the pairs were preceded by an unrelated prime. In the second list, the consistent-inconsistent target pairs that were paired with a related prime in the first list were now paired with an unrelated prime, and vice versa. Each list also included 28 filler trials. The targets of the filler trials were consistent or inconsistent monosyllabic words that began with a single nonplosive consonant $(/ \mathrm{s} /, / \mathrm{S} /, / \mathrm{w} /, / 1 /$, or $/ \mathrm{r} /$ ), and none had the same body as any of the targets of the test trials. Half of the fillers trials were preceded by a related prime, and the other half were preceded by an unrelated prime. 
Table 1

Mean Response Latency, Onset Duration, Rime Duration (in Milliseconds), and Percent Errors in Each Condition (With Standard Errors)

\begin{tabular}{|c|c|c|c|c|c|c|c|c|}
\hline \multirow[b]{2}{*}{ Condition } & \multicolumn{2}{|c|}{ Response Latency } & \multicolumn{2}{|c|}{ Onset Duration } & \multicolumn{2}{|c|}{ Rime Duration } & \multicolumn{2}{|c|}{$\%$ Error } \\
\hline & $M$ & $S E$ & $M$ & $S E$ & $M$ & $S E$ & $M$ & $S E$ \\
\hline \multicolumn{9}{|l|}{ Consistent } \\
\hline Unrelated & 387 & 7.8 & 104 & 3.9 & 354 & 10.4 & 3.4 & 1.1 \\
\hline Related & 382 & 7.4 & 103 & 3.9 & 353 & 10.7 & 4.6 & 1.0 \\
\hline \multicolumn{9}{|l|}{ Inconsistent } \\
\hline Unrelated & 397 & 7.9 & 118 & 5.1 & 358 & 10.2 & 11.2 & 1.8 \\
\hline Related & 386 & 7.5 & 107 & 4.6 & 360 & 10.9 & 4.8 & 1.2 \\
\hline
\end{tabular}

The first 2 trials were always filler trials. There were also 15 practice trials that approximated the same proportion of plosive, nonplosive, consistent, and inconsistent targets as well as the same proportion of related and unrelated trials as did the test and filler trials. None of the practice targets had the same body as the test and filler targets

\section{Apparatus}

The experiment was controlled by an IBM PC-compatible $486 \mathrm{CPU}$ computer. A 2-sec $11025 \mathrm{~Hz}$ digital sample of each naming response was recorded using a 16-bit SoundBlaster sound card and a Sensheimer super-cardiod headset microphone.

\section{Procedure}

Each trial proceeded as follows: a "Ready?" prompt along with a reminder to say "uuhhh" appeared in the center of the screen. (The subjects were specifically instructed to start saying "uuhh" immediately after initiating a trial and to continue saying "uuhhh" right up to the point at which they named the target word.) A trial was initiated by pressing a key on the keyboard. A centered "*" then replaced the "Ready?" prompt for $500 \mathrm{msec}$. Immediately after this, the prime word appeared in uppercase for $500 \mathrm{msec}$ in the center of the screen, replacing the "*." Finally, the target word appeared in lowercase for $2 \mathrm{sec}$ in the center of the screen, replacing the prime. The "Ready?" prompt then appeared once again.

Digital sampling began from the onset of the target stimulus on the CRT and continued for $2 \mathrm{sec}$. The audio sample was then stored to disk for offline analysis, as is described in the Results section below.

\section{RESULTS}

Two independent judges listened to the naming responses and marked pronunciation errors, which included mispronunciations, incorrect word pronunciations, multiple responses, heavy stutters, as well as late or absent "uuhhh" vocalizations. There were no disagreements between the two judges. One item, caste, was eliminated because of a high number of errors (more than $40 \%$ ). Three subjects whose error rates exceeded $20 \%$, as well as 7 additional subjects whose responses exceeded the latency and duration cutoffs (described in detail below) on more than $20 \%$ of the trials, were also excluded. Of the 1,350 responses from the 50 remaining subjects, the erroneous responses ( 81 in all) were removed and analyzed separately. Only correct responses were used in determining mean latencies and durations.

An audio analysis algorithm was then applied to the digitized responses to determine the offset of acoustic energy corresponding to the "uuhhh" vocalization at the beginning of each correct response. A 10-msec "analysis window" started at the beginning of the recording and cal- culated the mean absolute amplitude and number of sign changes in the slope of the waveform within the window. If both measures fell below a predetermined threshold, the algorithm recursively split and reanalyzed the window to more precisely locate the offset of acoustic energy. Otherwise, the algorithm moved the window forward through the recording and recalculated the signal detection measures. After the algorithm determined the vocalic offset (i.e., the beginning of the silent gap), the end of the silent gap was then determined. The analysis window was repositioned $10 \mathrm{msec}$ forward to ensure that any trailing noise from the offset would be skipped. Another set of thresholds were then used to determine whether either speech detection measure exceeded threshold. If so, the algorithm pinpointed the signal onset within the window. Otherwise, it continued forward through the sample to more precisely determine the onset of acoustic energy that corresponded to the release of the word-initial plosive.

Finally, the end of the word was found by starting at the end of the sample and moving the "analysis window" backward through the sample until either the mean absolute amplitude or the number of changes in the sign of the slope of the waveform within the window reached a predetermined criterion. At that point, the algorithm recursively split and reanalyzed the window for a more precise determination.

We determined the criterion values by trial and error; we guessed at initial values for the criteria, tested the algorithm on some sample data, examined a graphic display of the waveform with the algorithm's estimate of the beginning of the acoustic response, adjusted the criteria, and repeated the process until we were satisfied with the estimates determined by the algorithm. The software used in the analysis is described in more detail elsewhere (Kello \& Kawamoto, 1998).

On the basis of the temporal measures obtained using the algorithm, an additional 91 responses were excluded from the latency analyses. Of these, 6 responses (all beginning with a voiced plosive and spoken by male subjects) were not included because the algorithm failed to detect a silent gap. This failure was due to the low-intensity voiced energy that was continuously present from the offset of the "uuhhh" to the release of the plosive. The remaining 85 responses were not included because they fell below or above predetermined low and high cutoff 
values. The low and high cutoffs for response latency were 200 and $1,000 \mathrm{msec}$, respectively; low and high cutoffs for onset duration were 20 and $400 \mathrm{msec}$, respectively; and low and high cutoffs for rime duration were 50 and $600 \mathrm{msec}$, respectively. The vast majority of the responses (approximately $95 \%$ ) were eliminated because they fell below the response latency low cutoff or above the rime duration high cutoff. Most of the responses that fell below the response latency low cutoff were due to subjects starting the "uuhhh" after the stimulus had already been presented. This type of error could not be detected during the error-coding stage because coders could not determine when the stimulus was presented relative to the beginning of the "uuhhh" that they heard when they listened to the response. Most of the responses that fell above the rime duration high cutoff were due to noise (e.g., coughing or loud exhalation) after the word had been pronounced.

The mean response latency, onset duration, rime duration, and percentage of errors in each of the conditions are shown in Table 1 with their standard errors. Two-way analyses of variance, with regularity and prime as the two factors and with the above measures as the dependent variables, were carried out. Analyses with both subjects and items as the random factors were carried out. The consistency factor was within subjects but between items, and the prime factor was within subjects and items.

\section{Response Latencies}

The response latencies for targets preceded by a related prime were $8 \mathrm{msec}$ shorter than those for targets preceded by an unrelated prime, and the response latencies for consistent targets were $6 \mathrm{msec}$ shorter than those for inconsistent targets. The main effect of prime was significant $[F(1,49)=9.70, p<.005$ by subjects, and $F(1,25)=5.75$, $p<.05$ by items]. The main effect of consistency was significant by subjects $[F(1,49)=6.86, p<.05]$ but not by items $(F<1)$. The interaction of prime and consistency was not significant $(F \mathrm{~s}<1)$.

\section{Onset Durations}

The onset durations for targets preceded by a related prime were $6 \mathrm{msec}$ shorter than those for targets preceded by an unrelated prime, and the onset durations for consistent targets were $9 \mathrm{msec}$ shorter than those for inconsistent targets. The main effect of prime was significant $[F(1,49)=6.24, p<.05$ by subjects, $F(1,25)=$ $9.75, p<.005$ by items]. The main effect of consistency was significant by subjects $[F(1,49)=12.68, p<.001]$ but not by items $[F(1,25)=2.26, p>.14]$. The interaction of prime and consistency was significant by subjects $[F(1,49)=4.30, p<.05]$ and marginally significant by items $[F(1,25)=3.74, p<.07]$.

\section{Rime Durations}

The rime durations for targets preceded by a related prime were nearly identical to those for targets preceded by an unrelated prime. The main effect of prime and the interaction of prime and consistency were not significant ( $F \mathrm{~s}<1$ by subjects or items). We did not compare the rime durations for consistent and inconsistent targets, because the rimes were not identical.

\section{Errors}

There were fewer errors in the related condition than in the unrelated condition and fewer errors to consistent targets than to inconsistent targets. The main effect of prime was significant by subjects $[F(1,49)=4.21, p<.05]$ but not by items $[F(1,25)=2.57, p>.12]$. The main effect of consistency was significant by subjects $[F(1,49)=8.65$, $p<.005]$ but not by items $(F<2)$. The interaction of prime and consistency was significant by subjects $[F(1,49)=$ $9.60, p<.005]$ and marginally significant by items $[F(1,25)$ $=4.21, p<.06]$.

\section{DISCUSSION}

The locus of processing difficulty in the speeded naming task was investigated by considering semantic priming and consistency of pronunciation, two factors that are often examined separately but which, to our knowledge, have only recently been examined in a factorial design (Cortese, Simpson, \& Woolsey, 1997; Hess \& Foss, 1994). To provide a more comprehensive picture of the locus of processing difficulties, onset and rime durations as well as response latencies were measured.

We begin by considering the effect of semantic priming on onset and rime durations, the primary objective of our study. The most important results are that (1) onset durations are shorter in the related condition than in the unrelated condition but (2) rime durations are equal in the related and unrelated conditions. Of the proposals considered in the introduction, only the parallel phoneme activation scheme with a phonemebased motor program can account for both results. We note that this proposal can also account for the larger priming effect on onset duration for inconsistent words, as compared with consistent words. Thus, the effect of a semantic prime on duration appears to arise during the pronunciation generation stage rather than during a stage following it. These results also provide evidence that phonemes are activated in parallel rather than sequentially from left to right and that motor programs are based on the phoneme rather than the syllable. We do caution, however, that our results are based on subjects naming target words presented visually in prime-target pairs in the standard semantic priming paradigm; there may very well be a role for a cooperative account in normal speech.

These results, showing that the effects of a semantic prime on naming duration are localized to the onset, are important because both of the proposals put forth by Balota et al. (1989), the cooperative account and the sequential phoneme activation scheme with a phoneme-based motor program, predict an effect of the prime on rime duration. These results are also important because most models of word naming (Coltheart, Curtis, Atkins, \& Haller, 1993; Plaut, McClelland, Seidenberg, \& Patterson, 1996; Seidenberg \& McClelland, 1989) assume that pronunciation is initiated only after all the phonemes of a syllable or word become available (i.e., a syllable-based motor program), whereas we argue that the duration results can only be accounted for by models in which pronunciation is articulated as soon as the initial phoneme becomes available (i.e., a phoneme-based motor program)

We next consider response latency, focusing on the result that has recently attracted some attention, the interaction of prime and consistency. There are two reports of an interaction of prime and regularity (a factor closely related to consistency) on response latency, using the standard semantic priming paradigm (Cortese et al., 1997; Hess \& Foss, 1994). By contrast, we found no interaction of prime and consistency on response latency such as would be predicted by the parallel model 
with a phoneme-based motor program. We believe the reason for the discrepancy can be attributed to the different indices of response latency that were used in the different studies. In our study using the postvocalic naming task, the measure of response latency was vocalic offset latency, the latency of the offset of the "uuhhh." By contrast, in the Cortese et al. and the Hess and Foss studies using the standard naming task, the measure of response latency was naming latency, the latency of the onset of acoustic energy. The problem with naming latency, as we discussed in the introduction, is that it conflates response latency and initial phoneme duration for words beginning with plosives, because acoustic energy is generated only at the end of a plosive. Given that we do find an interaction of frequency and consistency for onset durations (i.e., initial phoneme durations), the prime $\times$ regularity interaction on naming latency might actually be due to onset duration effects rather than to response latency effects. This conflation of response latency and initial phoneme duration would also explain why Cortese et al. found a larger interaction when only words beginning with plosives were used (Experiment 2 ) than when words beginning with plosives and nonplosives were used (Experiment 1 ).

We close by noting the importance of avoiding the conflation of dependent variables. For example, naming duration of monosyllabic word conflates onset duration and rime duration, and naming latency of words beginning with plosive consonants conflates response latency and initial phoneme duration. The use of unconflated variables is particularly important in attempts to determine the locus of a variable's effect on speeded naming--both in terms of the stage of processing in which effects occur and in terms of the part of the pronunciation that is affected

\section{REFERENCES}

Balota, D. A., Boland, J. E., \& Shields, L. W. (1989). Priming in pronunciation: Beyond pattern recognition and onset latency. Journal of Memory \& Language, 28, 14-36.

BECKER, C. A. (1979). Semantic context and word frequency effects in visual word recognition. Journal of Experimental Psychology: Human Perception \& Performance, 5, 252-259.

Coltheart, M., Curtis, B., Atkins, P., \& Haller, M. (1993). Models of reading aloud: Dual-route and parallel-distributed-processing approaches. Psychological Review, 100, 589-608.

Cortese, M. J., Simpson, G. B., \& Woolsey, S. (1997). Effects of association and imageability on phonological mapping. Psychonomic Bulletin \& Review, 4, 226-231.

DELL, G. S. (1986). A spreading-activation theory of retrieval in sentence production. Psychological Review, 93, 283-321.

DELL, G. S. (1988). The retrieval of phonological forms in production: Tests of predictions from a connectionist model. Journal of Memory \& Language, 27, 124-142.

FowLER, C. A., \& HousUm, J. (1987). Talkers' signaling of "new" and "old" words in speech and listeners" perception and use of the distinction. Journal of Memory \& Language, 26, 489-504.

Grice, H. P. ( 1975). Logic and conversation. In P. Cole \& J. L. Morgan (Eds.), Syntax \& semantics: Vol. 3. Speech acts (pp. 41-58). New York: Academic Press.

Hess, D. J., \& Foss, D. J. (1994, November). The influence of contex on the regularity effect. Paper presented at the 35 th Annual Meeting of the Psychonomic Society, St. Louis.
Kawamoto, A. H., Kello, C. T., Jones, R. M., \& Bame, K. A. (1998). Initial phoneme versus whole word criterion to initiate pronunciation: Evidence based on response latency and initial phoneme duration. Journal of Experimental Psychology: Learning, Memory, \& Cognition, 24, 862-885.

Kello, C. T., \& Kawamoto, A. H. (1998). Runword: An IBM-PC software package for the collection and acoustic analysis of speeded naming responses. Behavior Research Methods, Instruments, \& Computers, 30, 371-383.

KUČERA, H., \& FrANCIS, W. (1967). Computational analysis of presentday American English. Providence, RI: Brown University Press.

LieBerman, P. (1963). Some effects of semantic and grammatical context on the production and perception of speech. Language \& Speech, 6, 172-187.

Plaut, D. C., McClelland, J. L., Seidenberg, M. S., \& Patterson, K. (1996). Understanding normal and impaired word reading: Computational principles in quasi-regular domains. Psychological Review, 103, 56-115.

SeidenberG, M. S., \& MCClelland, J. L. (1989). A distributed, developmental model of word recognition and naming. Psychological Review, 96, 523-568.

Shields, L. W., \& Balota, D. A. (1991). Repetition and associative context effects in speech production. Language \& Speech, 34, 47-55.

TARABan, R., \& MCCLELland, J. L. (1987). Conspiracy effects in word pronunciation. Journal of Memory \& Language, 26, 608-631.

\section{APPENDIX}

Targets and Primes Used in the Experiment

\begin{tabular}{lllllll}
\hline \multicolumn{3}{c}{ Consistent } & & \multicolumn{3}{c}{ Inconsistent } \\
\cline { 1 - 2 } \cline { 5 - 6 } Target & Related & Unrelated & & Target & Related & Unrelated \\
\hline bail & PAROLE & PASTEL & & bead & NECKLACE & NORTHERN \\
bake & COOK & COAT & & bowl & DISH & DAWN \\
boil & SIMMER & SAILOR & & bull & COW & CAR \\
bolt & WRENCH & WREATH & & bush & TREE & TRAP \\
cane & STICK & SCRUB & & caste* & CLASS & CRAWL \\
coop & CAGE & COST & & comb & HEADBAND & HUNDRED \\
curse & SWEAR & SWORD & & cough & SNEEZE & SLAVE \\
dutch & SWISS & SWEEP & & doll & TOY & TOWN \\
gale & GUST & GOWN & gild & PLATED & PLIERS \\
gorge & RAVINE & REPORT & & guise & MASK & MOSS \\
pack & GROUP & GLASS & & pear & APPLE & ANKLE \\
pet & CAT & CAB & & pint & QUART & QUEEN \\
pond & LAKE & LIST & pour & SPILL & SMOKE \\
tee & GOLF & GONG & ton & KILOGRAM & KEROSENE \\
\hline
\end{tabular}

* Caste was excluded because of high error rates.

(Manuscript received April 10, 1997; revision accepted for publication February 26, 1998.) 